\title{
Unblocking in a runway discrimination problem: The effects of a surprising shift from small reward to nonreward in $\mathrm{S}$ - relative to small reward throughout training
}

\author{
STEVEN J. HAGGBLOOM \\ Arkansas State University, State University, Arkansas
}

\begin{abstract}
Blocking and unblocking groups were trained in Phase 1 on an instrumental discrimination task in which internal cues were relevant and brightness cues were irrelevant. Both groups received large reward in S+ and small reward $(S)$ in $\mathbf{S}-$ in Phase 1. In Phase 2, brightness cues and internal cues were simultaneously and equally relevant for all groups. Group Blocking continued to receive $S$ on $S-$ trials in Phase 2, and learning about brightness was substantially reduced relative to that of appropriate control groups. Group Unblocking experienced a shift from $\mathrm{S}$ to nonreward $(\mathrm{N})$ in $\mathrm{S}$ - at the beginning of Phase 2 and learned as much about brightness as did the control groups.
\end{abstract}

The acquisition of discriminative stimulus control by internal, reward-produced cues in instrumental learning blocks (cf. Kamin, 1969) the acquisition of control by subsequently added relevant, but redundant, brightness cues (Capaldi, Verry, \& Nawrocki, 1982; Haggbloom, 1981). Haggbloom (1984a, 1984b) has reported that this blocking effect is greatly attenuated, a phenomenon called unblocking, by a surprising reduction in $\mathrm{S}-$ reward magnitude. In those experiments, the unblocking groups experienced small reward (S) on trials to the negative (S-) discriminandum in Phase 1 and then a sudden shift to nonreward $(\mathrm{N})$ on $\mathrm{S}$ - trials in Phase 2 . Blocking groups received $\mathrm{N}$ on all $\mathrm{S}-$ trials in both phases of training, and a control group, trained only in Phase 2, received $\mathrm{N}$ on all $\mathrm{S}-$ trials. Thus, the blocking, unblocking, and control groups were equated on postshift reward conditions. With this experimental design, the conclusion that blocking was attenuated by the shift from $\mathrm{S}$ to $\mathrm{N}$ in $\mathrm{S}$ - rests on the inference that a blocking effect would have occurred if subjects in the unblocking group had continued to receive $\mathrm{S}$ in $\mathrm{S}$ - during Phase 2 . The purpose of the experiment reported here was to test the validity of that inference.

The present experiment investigated unblocking by equating groups on preshift reward conditions, a design that is characteristic of unblocking experiments in Pavlovian conditioning. In Phase 1 , Groups Blocking and Unblocking were trained on a successive go/no-go discrimination problem on which reward-produced cues and certain intertrial-interval(ITI)-related cues were

This experiment was supported by an Arkansas State University faculty research grant to the author. The author's mailing address is: Department of Counselor Education and Psychology, P.O. Box 2127, State University, AR 72467-2127. relevant discriminanda (see Haggbloom, 1981). Those cues are identified collectively as internal cues to distinguish them from brightness cues. Brightness cues were constant across trials in Phase 1 and thus were irrelevant. Both groups received large reward (L) on trials to the positive (S+) discriminandum and $\mathrm{S}$ on $\mathrm{S}$ trials.

In Phase 2, internal cues and brightness cues were simultaneously and equally relevant for all groups. Groups Control/S and Control/ $\mathrm{N}$ began training in Phase 2. Groups Blocking and Control/S received $S$ on $\mathrm{S}-$ trials, and Groups Unblocking and Control/ $\mathrm{N}$ received $\mathrm{N}$ on $\mathrm{S}-$ trials. All groups received $\mathrm{L}$ on $\mathrm{S}+$ trials.

The acquisition of stimulus control by brightness was assessed, as in the experiments reported by Haggbloom (1981, 1984a, 1984b), by an opposed-cue test involving the reversal of brightness cues.

\section{METHOD}

\section{Subjects}

The subjects were 40 male rats, approximately 90-days old at the beginning of training, bred in the laboratory from Holtzman stock.

\section{Apparatus}

The apparatus consisted of two parallel straight alleys $105 \mathrm{~cm}$ long $\times 9 \mathrm{~cm}$ high and wide. The walls and floor of one alley were painted black, and the walls and floor of the other alley were painted white. The last $25 \mathrm{~cm}$ of each alley constituted a goalbox separated from the rest of the alley by a manually operated guillotine door. The doors and goalboxes were painted the same color as the alley in which they were located. Each goalbox contained an unpainted wooden goal cup. A gray startbox, $9 \mathrm{~cm}$ high and wide $\times 25 \mathrm{~cm}$ long, could be aligned to permit entry into one alley or the other. The startbox had a gray, manually operated guillotine door. 
The alleys were divided into three sections over which running times were recorded (respectively, start, run, and goal time). Start times were recorded from the opening of the startbox door, which triggered a $.01-\mathrm{sec}$ clock, to a point $30 \mathrm{~cm}$ into the alley. Run and goal times were recorded over the next 40 and $30 \mathrm{~cm}$, respectively. The offset of the first clock and the operation of the remaining clocks were controlled by photoelectric circuitry.

\section{Procedure}

The rats were housed in individual cages and had free access to food and water for at least 2 weeks prior to the 1 st day of food deprivation. The food-deprivation schedule consisted of $12 \mathrm{~g}$ of Wayne Lab Blox per day and was begun 14 days prior to the start of discrimination training. It continued throughout the experiment; the amount of food consumed in the runway during training was subtracted from the $12-\mathrm{g}$ daily ration. Water was always available.

On each of Days 12-14 of deprivation, the rats were handled in squads of four for 4 min per squad. After being handled on each day, the rats received 1045 -mg Noyes pellets in their home cages. Discrimination training began on Day 15 of deprivation.

On all rewarded trials, the rats were removed from the goalbox after the times had been recorded (after approximately $10 \mathrm{sec}$ ) unless the reward had not yet been consumed. Reward consisted of $1245-\mathrm{mg}$ Noyes pellets on $L$ trials and 2 pellets on $\mathrm{S}$ trials. On $\mathrm{N}$ trials, the rats were confined to the unbaited goalbox for $20 \mathrm{sec}$.

The rats were run in squads of four, containing two rats from each group during Phase 1 and one rat from each group during Phase 2 and the test phase. Within each squad, the order of administering trials was randomized daily. The order of successive squads was held constant across days.

\section{Design}

Only Groups Blocking and "Unblocking were trained in Phase 1 . Both groups were treated identically and received three consecutive L trials, separated by a 3-min ITI, followed $20 \mathrm{~min}$ later by three consecutive $\mathrm{S}$ trials, also separated by a 3-min ITI (LLL ... SSS schedule). On this schedule, stimuli unique to Trial 1 , and the memory of $\mathrm{L}$ produced on Trials 2 and 3 , function as $S+$ cues signaling $L$ on Trials 1,2 , and 3. Similarly, stimuli unique to the relatively long within-day ITI, and the memory of $S$ produced on Trials 3 and 4 , function as $S-$ cues signaling $S$ on Trials 4,5 , and 6 . The LLL ... SSS schedule defines a situation in which internal cues are relevant discriminanda (Haggbloom, 1981, 1984a). One-half of the rats in both groups received all of their Phase 1 trials in the black runway, and the remaining rats were trained in the white runway. Thus, brightness was irrelevant in Phase 1 . There were 10 days of training in Phase 1.

In Phase 2, Group Control/N began training and, along with Group Unblocking, received three consecutive $L$ trials, separated by a 3-min ITI, followed $20 \mathrm{~min}$ later by three consecutive $\mathbf{N}$ trials, also separated by a 3-min ITI (LLL ... NNN schedule). Group Control/S also began training in Phase 2 and, along with Group Blocking, received the LLL ... SSS schedule daily. Brightness cues were made relevant for all four groups in Phase 2. For one-half of the rats in each group, all L trials were administered in the black runway and all $S$ (or $N$ ) trials were administered in the white runway. These conditions were reversed for the remaining rats in each group. The newly added brightness cue became part of the $S-$ stimulus compound in each case. There were 8 days of training in Phase 2 .

A single, opposed-cue test day, on which brightness cues were reversed, followed Phase 2. Except for the reversal of brightness cues, all other procedures on the test day were the same as in Phase 2.

\section{RESULTS}

Start, run, and goal times were summed for each trial to obtain a total time, and all time scores were converted to speeds in centimeters per second. Only total speeds, which were representative of responding in each alley section, are reported here. Some analyses were performed on difference scores obtained by subtracting mean daily $\mathrm{S}-$ speeds from mean daily $\mathrm{S}+$ speeds.

\section{Phase 1}

Groups Blocking and Unblocking both ran faster in $\mathrm{S}+$ than in S- by the end of Phase 1 (data not shown). An analysis of variance (ANOVA) with groups (2) and brightness (2) as between-subjects variables and discriminanda (2), trials (3), and days (4) as within-subjects variables was applied to speeds over the last 4 days of Phase 1. Simple effects of discriminanda at each group showed that, for Groups Blocking and Unblocking, respectively, speed of running in S+ (means $=75.94$ and $81.82 \mathrm{~cm} / \mathrm{sec}$ ) were reliably faster than in $\mathrm{S}-($ means $=$ 48.11 and $62.39 \mathrm{~cm} / \mathrm{sec})[\mathrm{Fs}(1,16)=64.08$ and 31.18 , ps $<.001]$.

\section{Phase 2 and Test Day}

Figure 1 shows the mean difference between speed of running in S+ and S- collapsed over the last 4 days of Phase 2 and on the test day for all four groups. As can be seen, there was essentially no difference among the four groups in degree of differential responding to $\mathrm{S}+$ and $\mathrm{S}-$. The reversal of brightness cues substantially disrupted discriminative responding in both control groups and in Group Unblocking, but had much less of an effect on discriminative behavior in Group Blocking.

An ANOVA identical to that applied to speeds on the

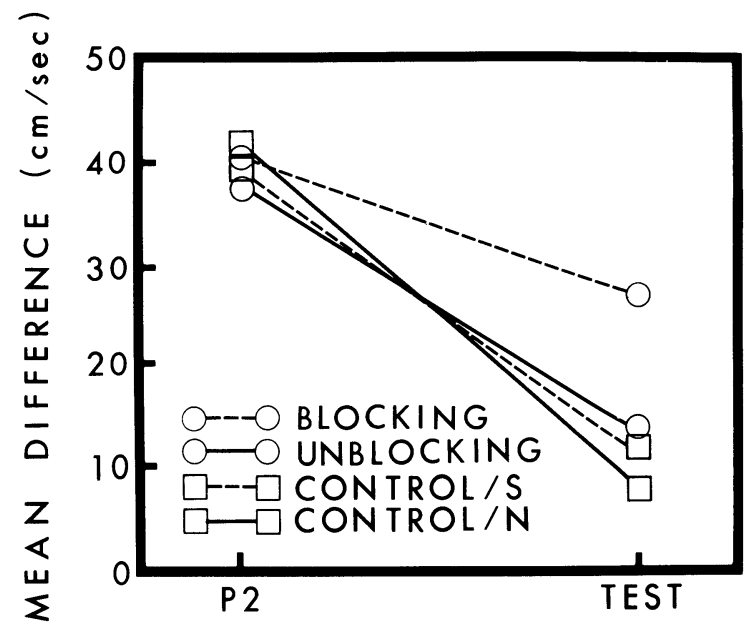

Figure 1. Mean difference between speeds in S+ and S- over the last 4 days of Phase 2 (P2) and on the test day (test) for each of the four groups. 
last 4 days for Phase 1, but now with four levels of groups, was applied to speeds on the last 4 days of Phase 2. Simple effects of discriminanda at each group showed that $\mathrm{S}+$ speeds were reliably faster than $\mathrm{S}-$ speeds for each group [smallest $F(1,32)=47.10, p<.001$, for Group Unblocking]. A similar ANOVA and simple effects showed that each group continued to run faster on $\mathrm{S}+$ trials than on $\mathrm{S}-$ trials on the test day [smallest $\mathrm{F}(1,32)=5.91$ for Group Control/N] .

An ANOVA applied to the difference between mean S+ and S- speeds on the last day of Phase 2 and the test day with groups (4) as a between-subjects variable and phase (2) as a within-subjects variable yielded a reliable groups $x$ phase interaction $[F(3,32)=3.33, p<.05]$. Simple effects of groups at each phase showed that the differences among groups over the last 4 days of Phase 2 (see Figure 1) were not reliable $[\mathrm{F}(3,32)=1.09]$ but that those on the test day were $[\mathrm{F}(3,32)=12.16$, $\mathrm{p}<.01]$. Simple effects of phase at each group showed that the reversal of brightness cues disrupted discriminative responding in each group [smallest $F(1,32)=7.04$, $\mathrm{p}<.05$, for Group Blocking]. Planned comparisons showed that discriminative responding on the test day was better in Group Blocking than in Group Control/S $[F(1,32)=4.57, p<.05]$ but that neither the difference between Group Unblocking and Group Control/N $[F(1,32)=1.16]$ nor that between Group Unblocking and Group Control/S $(\mathrm{F}<1)$ was reliable.

\section{DISCUSSION}

Groups Blocking and Unblocking were trained in Phase 1 with internal cues relevant and brightness cues irrelevant. Both of those groups and Groups Control/S and Control/N were trained with internal cues and brightness cues equally relevant in Phase 2. The acquisition of stimulus control by brightness cues in Phase 2 was substantially blocked in Group Blocking compared with Group Control/S. Blocking was prevented in Group Unblocking, which was shifted from $\mathrm{S}$ in $\mathrm{S}-$ during Phase 1 to $\mathrm{N}$ in $\mathrm{S}$ - during Phase 2. The results of this experiment indicate that, unless subjects receive a reward shift at the beginning of compound stimulus training, a blocking effect is produced by the Phase 1 training conditions experienced here by Groups Blocking and Unblocking and experienced by the unblocking groups employed by Haggbloom (1984a, 1984b). A previous experiment (Haggbloom, 1984b) demonstrated that the unblocking effect obtained under the present conditions is not a consequence of generalization decrement due to a change in reward-produced cues accompanying the reward shift.

\section{REFERENCES}

Capaldi, E. J., Verry, D. R., \& Nawrocki, T. M. (1982). Alley section effects on blocking. Bulletin of the Psychonomic Society, 20, 109-111.

HAGGBLOOM, S. J. (1981). Blocking in successive differential conditioning: Prior acquisition of control by internal cues blocks the acquisition of control by brightness. Learning and Motivation, 12, 485-508.

HAGGBLOOM, S. J. (1984a). Unblocking in a runway discrimination problem produced by a surprising reduction in $\mathbf{S}-$ reward magnitude at the beginning of compound stimulus training. Bulletin of the Psychonomic Society, 22, 63-66.

Hagabloom, S. J. (1984b). Unblocking in a runway discrimination problem produced by a surprising reduction in S- reward magnitude: The role of generalization decrement. Bulletin of the Psychonomic Society, 22, 469-471.

Kamin, L. J. (1969). Predictability, surprise, attention and conditioning. In B. Campbell \& R. Church (Eds.), Punishment and aversive behavior (pp. 279-296). New York: Appleton-CenturyCrofts.

(Manuscript received for publication May 25, 1984.) 